\title{
Biocontrol Treatments Confer Protection Against Verticillium dahliae Infection of Potato by Inducing Antimicrobial Metabolites
}

\author{
A. El Hadrami, L. R. Adam, and F. Daayf \\ University of Manitoba, Department of Plant Science, 222 Agriculture Building, Winnipeg, Manitoba, R3T 2N2, Canada
}

Submitted 28 April 2010. Accepted 18 November 2010.

\begin{abstract}
Verticillium wilt, caused by Verticillium dahliae Kleb., is a serious potato (Solanum tuberosum L.) disease worldwide, and biocontrol represents a promising eco-friendly strategy to reduce its impact. We used extracts from Canada milk vetch (CMV) and a set of four $V$. dahliae-antagonistic bacterial strains to coat potato seeds at planting and examined the degree of protection provided against $V$. dahliae as well as accumulation of soluble phenolics as markers for induced resistance. All tested treatments were effective in reducing disease severity, and CMV showed the highest level of protection. In this treatment, flavonol-glycoside rutin was a highly abundant compound induced in potato tissues, with levels two to three times higher than those detected in noninoculated controls and $V$. dahliae-inoculated plants. We investigated dose-dependent effects of rutin on $V$. dahliae growth and sporulation in vitro and in planta. The effect of rutin on mycelial growth was inconsistent between disk assay and amended medium experiments. On the other hand, significant reduction of $V$. dahliae sporulation in vitro was consistently observed starting at 300 and $100 \mu \mathrm{M}$ for isolates Vd-9 and Vd-21, respectively. We successfully detected 2protocatechuoylphloroglucinolcarboxylic acid (2-PCPGCA) using ultra-performance liquid chromatography tandem mass spectrometry, indicating that $V$. dahliae dioxygenally oxidizes quercetin. Quercetin, as an aglycone, is freed from the sugar moiety by glucosidases and rhamnosidases produced by the fungus and is a substrate for quercetinases. The occurrence of quercetinases in $V$. dahliae provides a background to formulate a hypothesis about how by-product 2-PCPGCA may be interfering with potato defenses.
\end{abstract}

Verticillium dahliae Kleb. is a serious soilborne fungal pathogen causing disease in many crops worldwide (Alkher et al. 2009) and increasingly emerging as a threat to potato production. In the absence of resistant cultivars, control strategies are limited to cultural practices such as crop rotation (Subbarao et al. 2007). However, the multiyear survival of $V$. dahliae resting structures (microsclerotia) in the soil and its ability to infect multiple hosts reduce the effectiveness of this practice. Alternative control methods include the use of fumigants (Fravel and Larkin 2000), green manures (Davis et al. 1996; El Hadrami et al. 2009a; Ochiai et al. 2007), amendments (Bailey and

Corresponding author: Fouad Daayf; Telephone: +1-204-474-6096; Fax: +1-204-474-7528; E-mail: Daayff@cc.umanitoba.ca

* The $\boldsymbol{e}$-Xtra logo stands for "electronic extra" and indicates that three supplementary figures are published online.
Lazarovits 2003), and biological control agents (MercadoBlanco et al. 2004; Paulin et al. 2009; Tjamos et al. 2004; Uppal et al. 2008). Fumigation is costly and can affect human health and the environment negatively. Green manures and organic amendments seem promising but have not provided consistent results. Nevertheless, these and other biological methods may reduce the impact of this disease if incorporated into an integrated pest management (IPM) strategy (Bailey and Lazarovits 2003). Implementation of an IPM strategy requires screening biocontrol agents and understanding their mechanisms of action (Uppal et al. 2007, 2008). Nonhost plant extracts can be a source of natural chemicals with a potential role in fungitoxicity or induction of plant defenses (Daayf et al. 1997; Uppal et al. 2008), for which secondary metabolites are suitable markers (Arfaoui et al. 2007; Daayf et al. 2003).

Higher plants accumulate a wide range of secondary metabolites, such as phenolics, saponins, and terpenes (Bennet and Wallsgrove 1994; Daayf et al. 2006; Osbourn 1996). Phenolic compounds and their glycosides are widely distributed in the plant kingdom and play important roles in cell protection (Daayf and Lattanzio 2008; Harborne and Williams 2000; Osbourn 1996). They can serve either as physical barriers between the stressed cells and their environment or as biochemical defenses against herbivores, pathogens, and ultraviolet radiation (Dixon and Paiva 1995; El Hadrami and Daayf 2009; El Hadrami et al. 2010; Ramos et al. 1997; Treutter 2006). Secondary metabolites with a flavonol or flavanone structure (i.e., rutin, quercetin, kaempferol and naringenin) (Supplementary Fig. 1) are naturally occurring antioxidants that accumulate in different plant tissues and play an important role in preventing cell oxidative injuries during plant defenses (Andersen and Markham 2006; Báidez et al. 2007; Treutter 2006). They can inhibit pathogen growth or oxidize into quinones, which can restrict microbial growth and development (Barry et al. 2002; Bennett and Wallsgrove 1994). Rutin (quercetin-3-rutinoside) is a flavonol glycoside that is widely present in fruit and vegetable crops and one of the first flavonoid signals released by host plant roots toward rhizobia (Phillips 1993) and mycorrizhal fungi (Lagrange et al. 2001) and during plant pruning and other processes that result in wounding. In their coevolution with specific or alternative hosts, pathogens have evolved mechanisms to degrade, detoxify, or tolerate such molecules. Degradation of flavonols such as quercetin is well-documented in bacteria (Braune et al. 2001). In fungi, such a phenomenon has been described, so far, in ascomycetes, i.e., Aspergillus spp. (Bartz 1971; Merkens et al. 2007; Steiner et al. 2002; Tranchimand et al. 2008; Westlake et al. 1961) and basidiomycetes (Sariaslani and Dalton 1989) but not in V. dahliae. For instance, Aspergillus flavus is known to activate glucosidases 
and esterases, such as rutinases when grown on rutin (quercetin 3-O-rhamnoglucoside)-rich media as the sole carbon source, and also an inducible dioxygenase called quercetinase. The latter is able to oxygenolytically cleave two carbon-carbon bonds from the heterocyclic ring of the rutin aglycone (quercetin) into carbon monoxide and 2-protocatechuoylphloroglucinolcarboxylic acid (2-PCPGCA) (Supplementary Fig. 2). The same reaction is thought to take place in Pullularia spp. (Oka and Simpson 1971), Fusarium spp. (Bartz 1971), and other Aspergillus spp. (Oka et al. 1971). This formation of carbon monoxide is peculiar to fungi, since most bacteria reduce flavonols instead of oxidizing them, with the exception of three prokaryotes that possess dioxygenases and do, in fact, release $\mathrm{CO}$ in the same way as fungal quercetinases (Wray and Abeles 1993).

In recent studies, we tested the efficacy of several biological treatments (beneficial bacteria and plant extracts) in reducing potato verticillium wilt in vitro, under growth-room conditions, and in the field for two seasons at various locations (Uppal et al. 2007, 2008). These studies allowed us to test various treatment application methods and to categorize them based on their mode of action (i.e., antagonism, antibiosis, enhancement of host defenses). The objective of the present study was to investigate the mechanisms by which Canada milk vetch (CMV) extracts induced potato defense responses against $V$. dahliae. Bearing in mind the ability of fungi to detoxify plant defense secondary metabolites and to interfere, pre- or posttranscriptionally, with host defense pathways (El Hadrami et al. 2009b), we investigated the response of $V$. dahliae to rutin and related metabolites produced by potato in response to this fungus. In order to elucidate potential mechanisms of degradation, we subsequently analyzed catabolic processes through which $V$. dahliae responds to these secondary metabolites.

\section{RESULTS}

\section{Potato protection against verticillium wilt} using biological treatments.

Disease severity was reduced in all inoculated plants pretreated with selected bacterial strains or CMV extract as compared with the inoculated control. CMV treatment showed the most significant effect in reducing verticillium wilt (Fig. 1). The observed effects correlated with the frequency of colonization of the tissues by $V$. dahliae detected by polymerase chain reaction (PCR) (Fig. 1).

\section{Soluble phenolics analysis in potato tissues}

after biological pretreatment, V. dahliae inoculation, or both.

As most selected biocontrol treatments were expected to act through induction of host resistance, soluble phenolics were examined as markers of such an induction. Flavonoids and hydroxycinnamates were the main families detected by highperformance liquid chromatography (HPLC), ultra-performance liquid chromatography (UPLC), and thin-layer chromatography (TLC) (Fig. 2) in potato tissues in response to infection by $V$. dahliae or treatment with different biological agents, especially CMV. The flavonol-glycoside rutin accumulated greatly in plants treated with CMV and then inoculated with $V$. dahliae (Fig. 2). Complementary analysis involving spiking and coelution with commercial rutin standard on TLC and HPLC along with UPLC-tandem mass spectrometry (MS/MS) analyses (Fig. 2C) confirmed the accumulation of rutin in potato plants treated with the tested biocontrol agents, especially CMV. Other flavonoids (apigenin, kaempferol, quercetin, and umbelliferone derivatives) were also detected but to a lower extent than rutin. Induced and accumulated hydroxycinnamates (Fig. 2A) included derivatives of caffeic, chlorogenic, and $p$-coumaric acids.
Both treatment with CMV and inoculation with $V$. dahliae have led to an accumulation of rutin twice the level of the healthy control (Fig. 2B). However, combining CMV treatment and inoculation resulted in an accumulation three times higher than that in the CMV-treated, noninoculated plants, almost four and two times the level of the healthy and inoculated controls, respectively (Fig. 2B). The other biological treatments did not show a significant increase in rutin accumulation (Fig. 2B). Rutin was not detectable in crude CMV extracts.

Based on regression analysis between rutin concentrations and vascular discoloration scores, variation of rutin concentration explains about $60 \%$ of the vascular discoloration variability observed. Considering only the inoculated treatments in the analysis showed a similar result, with $66 \%$ of the vascular discoloration variability explained by rutin content $(y=1.0754 x+$ $\left.1.1889 ; R^{2}=0.6635\right)$.

\section{Inhibition of $V$. dahliae growth and sporulation by rutin.}

Since rutin was the major soluble phenolic accumulated in either pretreated or inoculated plants or both, we tested its direct or indirect effect on $V$. dahliae grown in vitro, using a commercial standard. In addition, to determine the sporulation inhibition threshold, we assayed the growth of $V$. dahliae mycelia on solid media containing rutin concentrations of 0,100 , $300,600,900$, and $1,500 \mu \mathrm{M}$. Analysis of the effect of rutin on mycelial growth using paper disks showed $\mathrm{IC}_{50}$ values (concentration causing $50 \%$ inhibition) of $1.28 \pm 0.1$ and $1.00 \pm 0.2$ $\mathrm{mM}$ for $\mathrm{Vd}-21$ and $\mathrm{Vd}-9$, respectively, but the variability and low differences in growth inhibition did not allow calculation of $\mathrm{IC}_{50}$ values when rutin was directly incorporated into the culture medium. On the other hand, significant reduction of $V$. dahliae sporulation in vitro was observed starting at 300 and $100 \mu \mathrm{M}$ for isolates Vd-9 and Vd-21, respectively (Fig. 3). We further examined the interaction between $V$. dahliae and rutin in planta. Complementary experiments conducted on potato leaves, stem sections, and roots infiltrated with $75 \mu \mathrm{g}$ of rutin per milliliter and further inoculated with $V$. dahliae revealed an ability of the fungus to induce necrosis and grow in infiltrated tissues. Analysis of soluble phenolics from these tissues showed

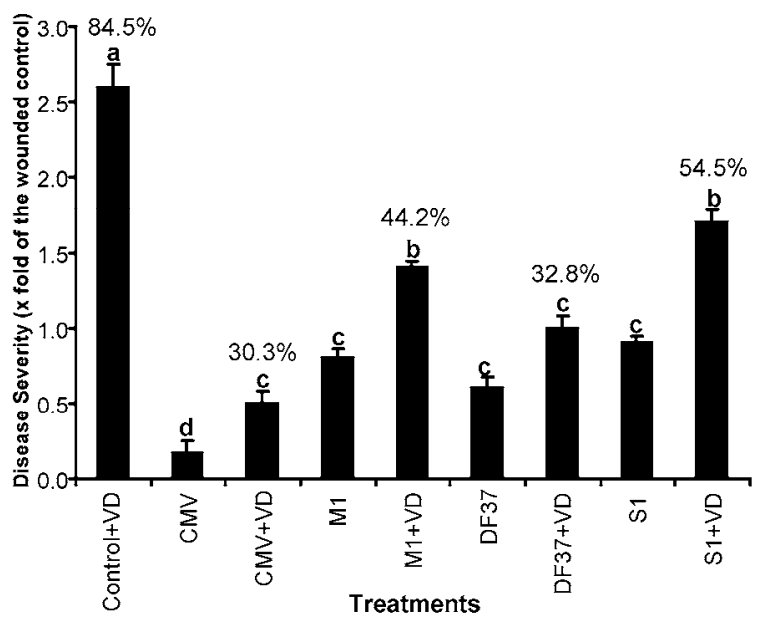

Fig. 1. Six-week postinoculation disease severity (ratio to the wounded noninoculated control) on potato plants pretreated, by 24 -h presowing seed coating, with Canada milk vetch plant extract (CMV) or different biocontrol agents, Bacillus pumilus (M1), Pseudomonas fluorescens biotype F (DF37), Bacillus sp. (S1), and inoculated (+VD) or not with Verticillium dahliae. As the same results were observed for both tested isolates, only data for Vd9 isolate is shown. Bars represent the standard error. Histograms within the same chart having the same letter do not differ significantly according to the Newman-Keuls test $(P<0.05)$. The numbers above the inoculated treatments represent the frequency of tissue colonization by V. dahliae according to polymerase chain reaction assays. 
a degradation of rutin and the appearance of by-products with characteristics recalling phloroglucinol derivatives (Fig. 4).

\section{Degradation of rutin and its derivatives by $V$. dahliae in vitro.}

After determining that $V$. dahliae was able to grow in vitro in the presence of rutin and that this flavonol-glycoside is degraded in its presence in planta, we ran a filter-paper disk assay and examined whether $V$. dahliae uses the whole molecule as a carbon source or only the sugar moiety. In either case, $V$. dahliae is left in the presence of a potentially toxic flavonol aglycone (quercetin). We then included rutin, its aglycone quercetin, a related flavonone, and phloroglucinol, given the nature of the by-product derivative detected in planta. The degradation of these compounds by $V$. dahliae was examined in sterile distilled water (SDW) or liquid media composed only of inorganic salts, 'minimum media', or Czapeck-Dox. In SDW and minimum media, $V$. dahliae isolates were unable to grow
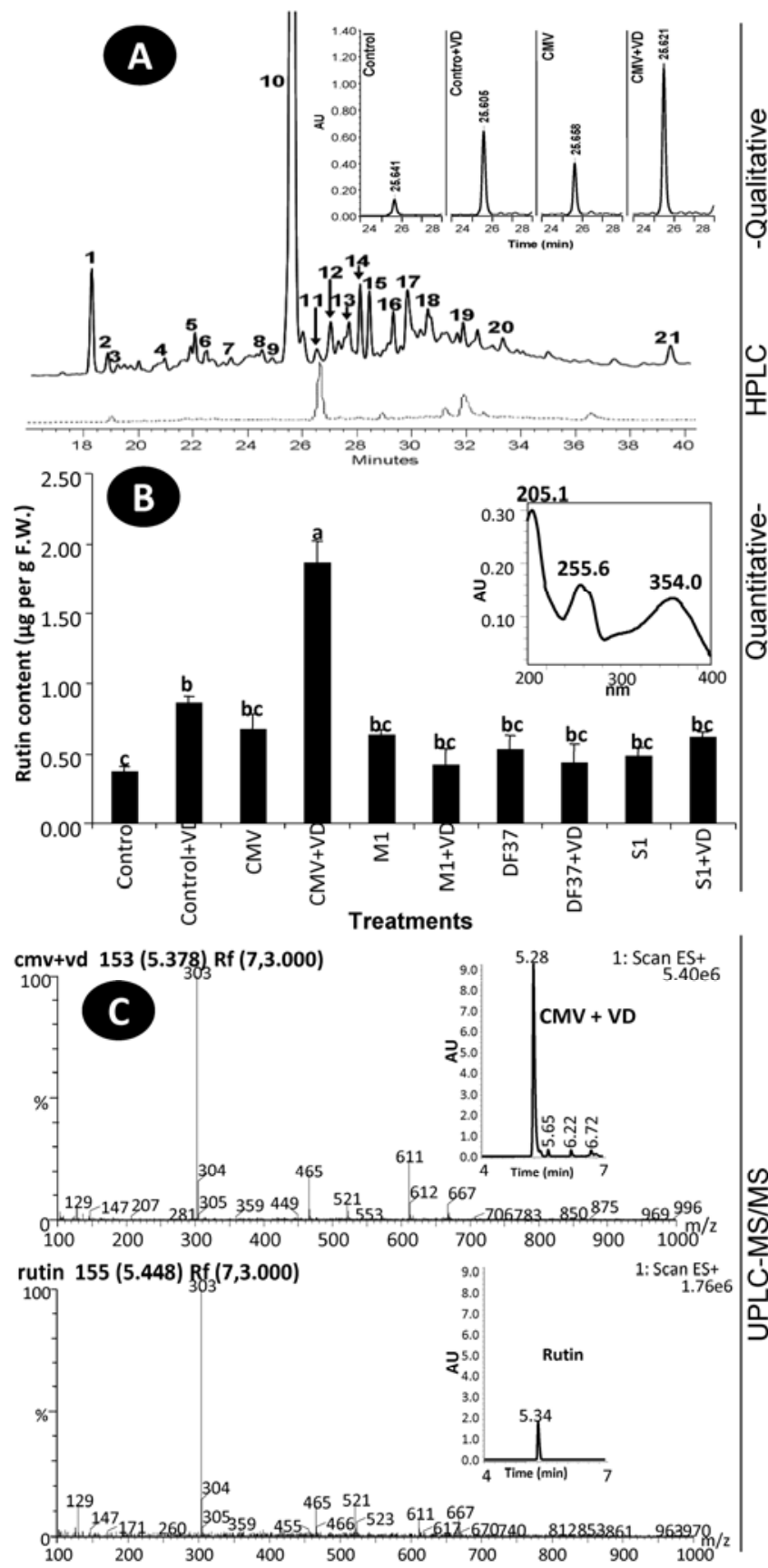

properly unless supplemented with either glucose, rutin, quercetin or naringenin, confirming the ability of both tested isolates to use these flavonols and flavanones as a carbon source. Czapeck-Dox, on the other hand, was more favorable for growth and development, and showed a decrease over time in the concentration of these flavonoids (up to four weeks after transfer) (Fig. 5). UPLC-MS/MS analysis revealed the presence of phloroglucinol derivatives such as 2-PCPGCA (Supplementary Fig. 3). Including phloroglucinol in liquid media did not affect the growth or development of the two tested $V$. dahliae isolate (Fig. 5).

\section{Confirmation of the $V$. dahliae-derived} quercetinase activity in vitro.

After predicting a number of parameters about the putatively secreted quercetinase protein, we wanted to confirm its enzymatic activity in vitro, and therefore, we proceeded with protein isolation and enzymatic assays. Examination of quercetinase

Fig. 2. Accumulation of rutin in response to biological treatments and inoculation with Verticillium dahliae. A, High-performance liquid chromatography (HPLC) (maxplot output) showing the soluble phenolics profile from (---) potato plants control and (-) plants treated with Canada milk vetch (CMV) extract and then inoculated with $V$. dahliae (isolate Vd9) The samples were from upper stems harvested 6 weeks after inoculation. Labeled peaks have been tentatively identified based on their retention time, characteristic UV spectra, coelution with commercial standards, and complementary thin-layer chromatography analysis. 1 and 8: methoxycinnamic acid derivatives; 2: apeginin derivative; 3 : unidentified; 4: chlorogenic acid derivative; 5 : caffeic acid derivative; $6: m$-coumaric acid derivative; 7: p-coumaric acid derivative; 9: benzoic acid derivative; 10: rutin; 11, 12, 13, and 15: unidentified flavonols; 14: kaempferol derivative; 16 : 4-methyl-umbelliferyl- $\beta$-glucoronide; 17,19 , and 20: 4-methyl-umbelliferone derivatives; 18: quercetin derivative; 21 : naringenin derivative. On the right side, four HPLC chromatograms illustrate the variation of rutin (peak 10) in treatments involving CMV. B, Bars show rutin accumulation (peak 10, measured in micrograms per gram of fresh weight [F.W.]) in tissues from nontreated (control) and biocontrol-treated potato plants in the presence or in the absence of $V$. dahliae isolate $\mathrm{Vd} 9$ (VD). Bars represent the standard deviations to the means. Histograms with the same letter do not differ significantly according to the Newman-Keuls test $(P<0.05)$. Right, the UV spectrum of rutin. C, Ultra-performance liquid chromatography and mass spectrometry profiles of a commercial standard (rutin) and the major induced compound in potato plants upon treatment with CMV extract and inoculation with $V$. dahliae isolate $\mathrm{Vd} 9(\mathrm{CMV}+\mathrm{Vd}) . \mathrm{m} / \mathrm{z}: 611$ represents rutin and the 303 its aglycone deprived of its sugars moiety (quercetin).

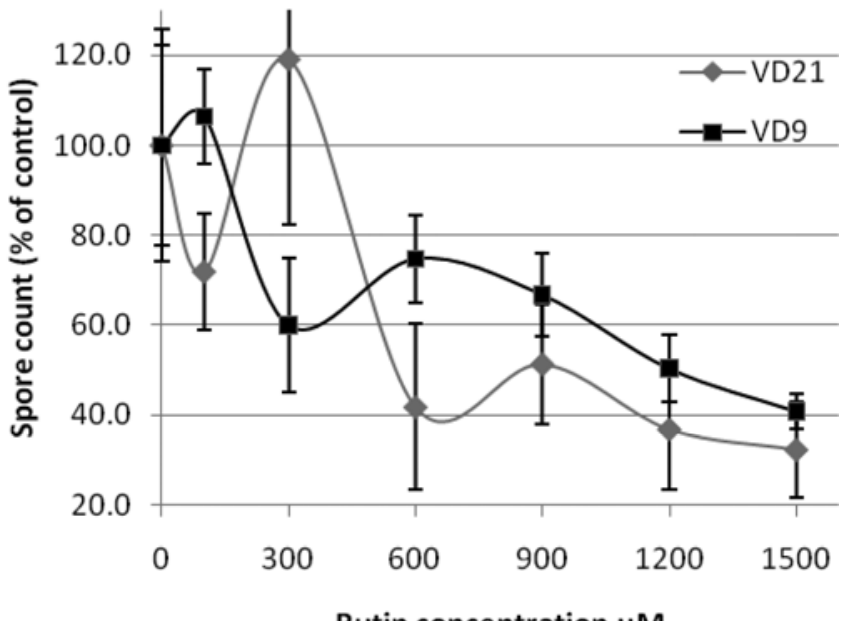

Rutin concentration $\mu \mathrm{M}$

Fig. 3. Reduction of spore production by Verticillium dahliae in response to increasing concentrations of rutin. 
activity revealed an enzymatic activity averaging 160 international units (IU) ( $1 \mathrm{IU}=1 \mu \mathrm{mol}$ quercetin per hour cleaved under standard conditions). The specific activity average was $2,516 \mu \mathrm{mol}$ quercetin per min per milligram of protein per gram of mycelium fresh weight (FW). This activity was abolished in the presence of either kojic acid or prohexadione-Ca, both known to be dioxygenase inhibitors.

\section{DISCUSSION}

An earlier study focusing on the effectiveness of several biocontrol agents in controlling verticillium wilt, when applied to potato seed-pieces at planting, revealed lower levels of disease in response to treatment with CMV extract, under both controlled and field conditions in various locations and growing seasons (Uppal et al. 2008). In the present study, we show this protection to occur along with a substantial accumulation of the flavonol glycoside rutin in treated potato tissues, whereas potato's inability to fight verticillium wilt in the other treatments, which involved beneficial bacteria, was associated with lower accumulation of rutin. Thus, rutin seemed to play a differential role, in a dose-response manner, in the response of potato to $V$. dahliae infection. However, defense-related sesquiterpenes such as rishitin and solavetivone have probably been induced by the tested treatments but have not been considered in this study.

Extracts from potato plants that were treated with certain biological agents prior to inoculation inhibited the growth of $V$. dahliae. Commercial rutin clearly reduced the rate of sporulation of both isolates. Previous papers had also clearly shown the inhibitory effects of rutin and quercetin on the growth of $V$. dahliae (Baidez et al. 2007). Our findings confirmed a direct

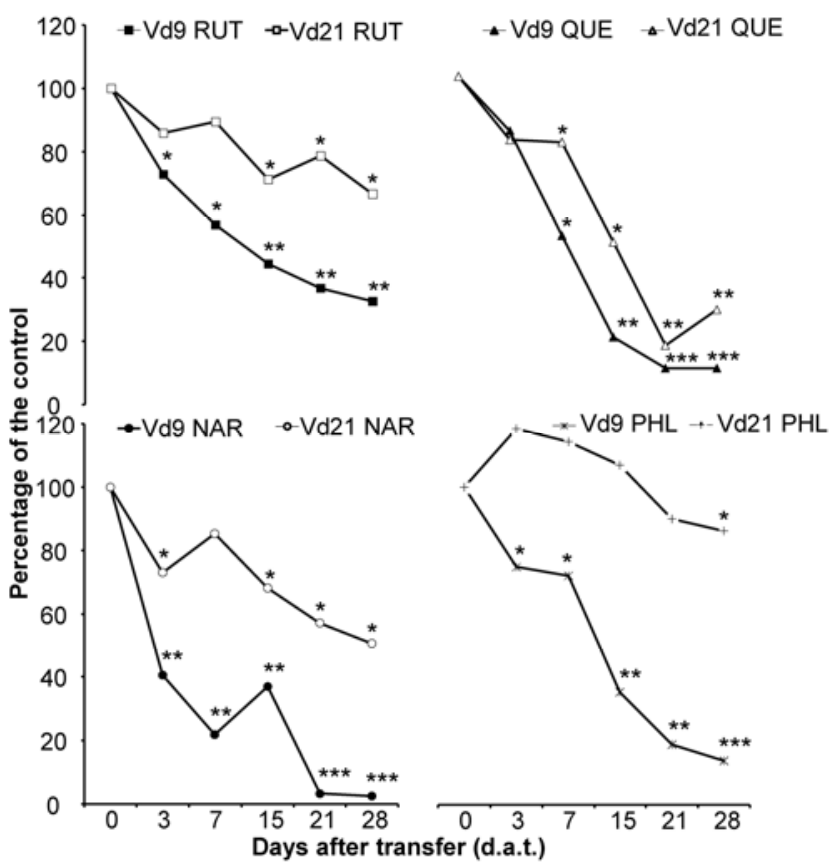

Fig. 5. Over time, variation of rutin (RUT), quercetin (QUE), naringenin (NAR), and phloroglucinol (PHL) content added to the Czapeck-Dox liquid media (commercial standard solutions of the compounds calibrated at $100 \mu \mathrm{g} / \mathrm{ml}$ ), relative to noninoculated control media, in the presence of two Verticillium dahliae isolates ( $\mathrm{Vd} 9$ and $\mathrm{Vd} 21)$. Every datapoint represents an average of three replicates, and statistical significance as compared with the initial time is shown with asterisks (* indicates $<0.05$; ** indicates $<0.1$; and $* * *$ indicates $<0.01)$.

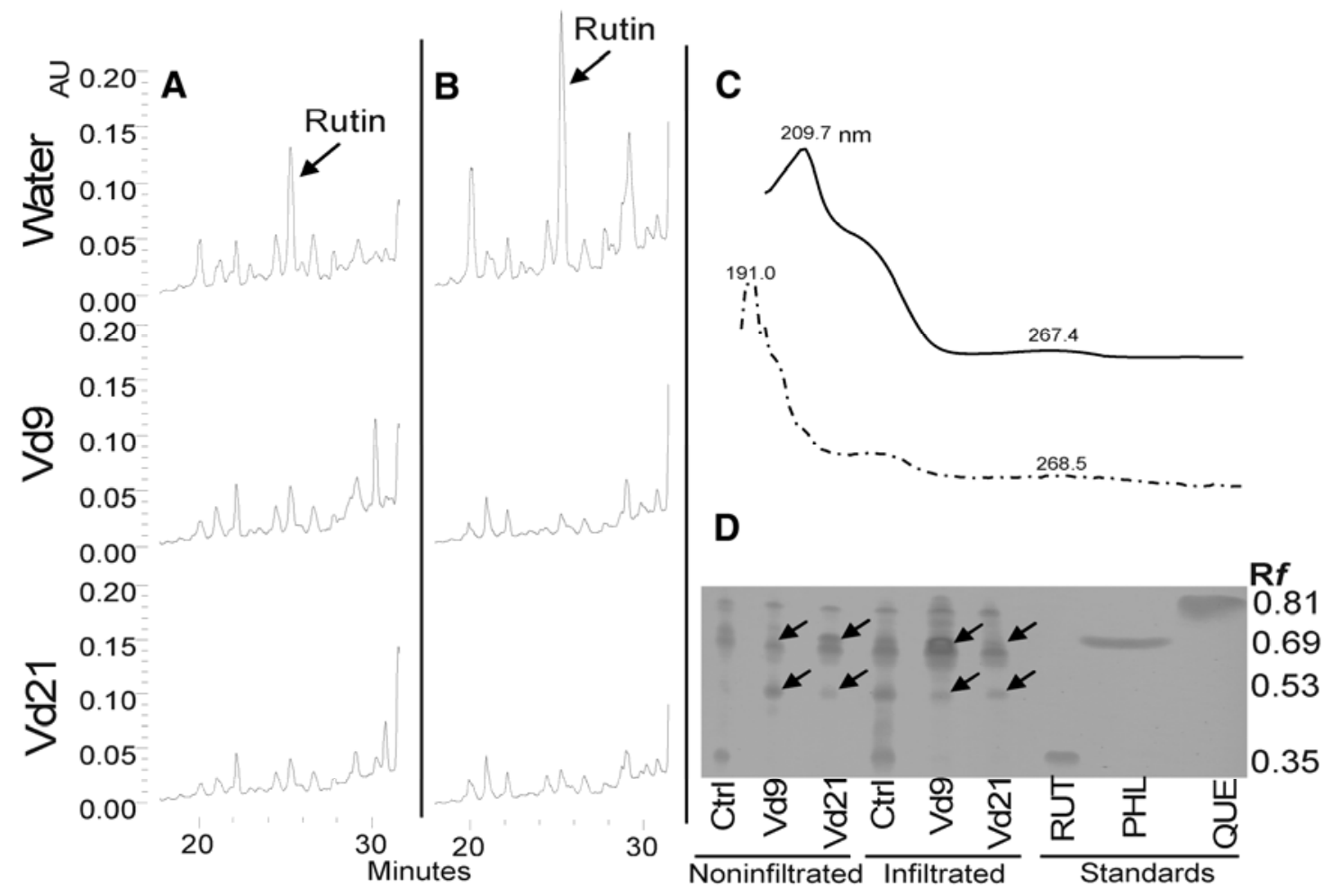

Fig. 4. A, High-performance liquid chromatography showing the soluble phenolic profile of potato leaves noninfiltrated or B, infiltrated with rutin and further inoculated with Verticillium dahliae isolates. C, shows the UV spectra of a commercial phloroglucinol standard (-) along with the phloroglucinol-

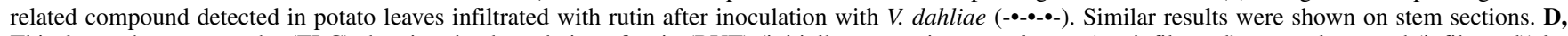
Thin-layer chromatography (TLC) showing the degradation of rutin (RUT) (initially present in potato leaves (noninfiltrated) or supplemented (infiltrated)) by two isolates of $V$. dahliae ( $\mathrm{Vd} 9$ and $\mathrm{Vd} 21$ ) and the accumulation of a by-product related to phloroglucinol (PHL) (i.e., 2-protocatechoylphloroglucinolcarboxylic acid). QUE = quercetin. $R_{\mathrm{f}}$ (retardation factor) values of the detected compounds on TLC are to the right. The arrows indicate the by-products that increased in intensity after inoculation or infiltration of rutin into the tissues. 
effect of the induced flavonol on the pathogen but also pointed to the induction of other defense metabolites that would complement such an inhibitory effect. They also showed the ability of $V$. dahliae to grow in presence of rutin, which suggested the ability of $V$. dahliae to metabolize this flavonol-glycoside. This ability is mainly due to this pathogen's glucosidases and rhamnosidases, which help cleave the sugar moieties from the flavonol-glycoside rutin, thereby freeing the quercetin aglycone. In addition, our results suggest that $V$. dahliae is also able to secrete, extra-cellularly, one or more quercetinases that detoxify the aglycone quercetin (unpublished), and additional characterization of the genes encoding these enzymes in $V$. dahliae revealed strong homology with well-characterized fungal and bacterial quercetinases. This gives $V$. dahliae the opportunity to counteract the rutin being produced by the plant in response to infection and supports earlier reports showing rutin as a quercetinase inducer in fungi (Tranchimand et al. 2008) due to its A ring.

Quercetinases, also known as extracellular 2,3- or 2,4-dioxygenases (EC 1.13.11.24 and EC 1.13.11.47, respectively), are a family of enzymes that are known to act on chromones such as the phenyl-benzopyrone skeleton, which possesses substituents like methyl or phenyl groups on carbon 2, a free hydroxyl on carbon 3, and a double bond between either carbons 2 and 3 or 2 and 4. By providing ${ }^{14} \mathrm{C}$-radiolabeled quercetin on $\mathrm{C}_{3}$ to these enzymes, Simpson and associates (1960) detected ${ }^{14} \mathrm{CO}$, while providing ${ }^{18} \mathrm{O}_{2}$ showed that the oxygen does not get incorporated into the carbon monoxide but is, rather, found in the carboxyl and the ester carbonyl groups of 2-PCPGCA. In the present study, $V$. dahliae isolates were able to grow in the presence of either rutin or its aglycone quercetin. This successful growth was ascribed to the secretion of an extracellular dioxygenase that cleaved quercetin and led to the accumulation of the byproduct 2-PCPGCA, which we effectively detected by UPLCMS/MS. Although no evaluation of CO content was conducted in this study, it is expected that the cleavage of quercetin would occur as described in other fungal species (Merkens et al. 2007; Steiner et al. 2002; Tranchimand et al. 2008).

Given that rutin is highly induced in potato in response to successful biocontrol agents and only weakly to the noneffective agents and its degradation by $V$. dahliae, the success of $V$. dahliae in the rhizosphere may be enhanced by its quercetinase activity. Such activity allows it to counter host plant defenses, i.e., synthesis and accumulation of flavonols and flavonones, by turning them into by-products such as 2-PCPGCA. When released, the phloroglucinol moiety may alter the equilibrium among microbial communities in the rhizosphere, thereby helping $V$. dahliae to better colonize it over other pathogens and antagonists. The protocatechuic moiety, on the other hand, could be converted into benzoate and salicylates, which can elicit the salicylic pathway in planta after infection. This elicitation may indirectly interfere with the potato defenses activated through the jasmonic acid (JA) pathway and is in line with the negative feedback between both pathways, as described by Kunkel and Brooks (2002) and Spoel and associates (2003). In a preliminary study, we showed that CMV produces jasmonaterelated compounds and may be mediating defenses that rely on the activation of the JA pathway in infected potato plants (El Hadrami et al. 2009a). Such elicitation of a given pathway (i.e., salicylic acid pathway) to interfere with the more adequate defense pathway (i.e., the JA pathway) is known in tobacco in response to Pseudomonas syringae (Rayapuram and Baldwin, 2007). A recent study (Thatcher et al. 2009) reported the ability of Fusarium oxysporum to hijack the COI1-mediated jasmonate signaling in order to promote disease development in Arabidopsis thaliana. The present study is in line with the occurrence of such a phenomenon in the potato- $V$. dahliae interaction. Further studies are needed to unravel the cross-talk among these and other pathways and investigate the role of $V$. dahliae quercetinases in the phenomenon. Also, it will be of interest to study functionally, by gene knock-out and overexpression, the role of such genes in the pathogenicity of this soilborne pathogen.

In the present study, we have also seen a significant correlation between vascular browning and rutin content. Vascular discoloration can be a sign of plant defense activation through enzymatic reactions involving oxido-reductases, such as polyphenol oxidases and peroxidases. These reactions take place in the woody xylem and involve both reaction and barrier zones (Barry et al. 2002), the former in the tissue damaged by the pathogen and the latter in cambium cells (Barry et al. 2002). Barrier zones are thought to be more resistant to fungal spread than reaction zones (Bauch et al. 1980). Further investigations are needed to examine the effect of rutin on $V$. dahliae in more detail and to determine the involvement of oxido-reductases in reducing disease and its progress towards the upper parts of the plant. Comparison of the response of cultivars with differential susceptibility or tolerance to a set of isolates with varying levels of aggressiveness will be a step forward to acquire such knowledge. It would be also interesting to screen $V$. dahliae isolates for the presence of quercetinase homologs to better understand the mechanisms by which this pathogen takes advantage of the low doses of rutin produced by the host. Meanwhile, the active ingredients of CMV that induce the observed systemic acquired resistance are under investigation, and the preliminary results indicate jasmonate derivatives are potential active ingredients in CMV.

$V$. dahliae possesses the ability to respond to the rutin elicited by biocontrol agents. Knowing that rutin can be systemically transported in planta (Buer et al. 2007), there seems to be a zigzag race between the host and the pathogen, leading to either an enhanced host defense or a counter-defense by the pathogen. Biocontrol agents could be efficient in reducing verticillium wilt in potato if they are able to induce this and other metabolites at levels high enough to restrict $V$. dahliae's development or if they act synergistically to fight the pathogen (Daayf et al. 1997). The mechanisms used by $V$. dahliae to interact with potato cultivars other than Russet Burbank remain to be investigated.

\section{MATERIALS AND METHODS}

\section{Plant material and growth conditions.}

Plants of the potato cultivar Russet Burbank, moderately susceptible to verticillium wilt, were grown from tuber seedpieces in $7.5-\mathrm{cm}$ diameter clay pots in a pasteurized soil-sand $\operatorname{mix}(1: 1, \mathrm{vol} / \mathrm{vol})$ at $20^{\circ} \mathrm{C}$ day and $16^{\circ} \mathrm{C}$ night and with a $16-\mathrm{h}$ photoperiod. They were hand watered daily, were fertilized (NPK, 20:20:20), and were treated with insecticides (i.e., antispider mites, antiaphids) when necessary.

\section{Inoculum preparation and inoculation procedure.}

Two highly aggressive isolates (Vd1396-9 [Vd-9] and Vd1398-21 [Vd-21]) were selected from a set of single-spore V. dahliae isolates previously tested for pathogenicity (Alkher et al. 2009; Uppal et al. 2007). These two isolates were recovered from potato tissues grown locally. Conidial suspensions $\left(10 \mathrm{ml}\right.$ at $10^{6}$ conidia per milliliter) were used to inoculate each plant by root dipping (Alkher et al. 2009).

\section{Biological treatments.}

The bacteria used in this study, namely Bacillus pumilus (M1), Pseudomonas fluorescens biotype F (DF37), and a $B a$ cillus sp. (S1) were previously isolated from the phyllophane 
and rhizosphere of potato and canola plants (Daayf et al. 2003; Uppal et al. 2007). They were chosen on the basis of either their inhibitory effect on V. dahliae growth (Uppal et al. 2007), their ability to enhance potato defense mechanisms (Daayf et al. 2003), or both. Bacterial suspensions were prepared by growing bacteria in $100 \mathrm{ml}$ of Difco nutrient broth (Becton Dickinson \& Co., Franklin Lakes, NJ, U.S.A.) for 2 days at room temperature under shaking $(200 \mathrm{rpm})$. The suspensions were then centrifuged at $3,000 \times g$ for $5 \mathrm{~min}$, and the pellet was transferred into SDW and was adjusted to $10^{8} \mathrm{CFU}$ per ml. Extracts from CMV (Astragalus canadensis L.) were prepared by grinding the leaves to powder in liquid nitrogen and transferring $30 \mathrm{~g}$ into an Erlenmeyer flask containing $100 \mathrm{ml}$ of $80 \%(\mathrm{vol} / \mathrm{vol})$ ethanol. After overnight shaking at $4{ }^{\circ} \mathrm{C}$ in the dark, the crude extracts were filtered through Whatman No. 1. Filtrates were diluted 10 times in SDW and were then used to coat seeds just before planting by mixing them with potato seed pieces ( $1 \mathrm{ml}$ for 40 to $80 \mathrm{~g}$ of seed pieces) in Ziploc plastic bags. Control tubers were treated with SDW containing an equivalent volume of ethanol.

\section{Disease rating.}

Disease rating was conducted over time ( 2 to 6 weeks after inoculation), using the area under disease progress curve (AUDPC) (Campbell and Madded 1990), calculated according to the following formula:

$$
\mathrm{AUDPC}=\sum_{\mathrm{i}=1}^{\mathrm{n}-1}\left[\left(\left(y_{i}+y_{i+1}\right) / 2\right)\left(t_{i+1}-t_{i}\right)\right]
$$

where $n$ is the total number of assessments in weeks, $y_{i}$ is the disease severity at the $i$ th assessment week, and the term $t_{i+1}-t_{i}$ is the time duration between two assessments. Vascular discoloration was assessed on 5-mm-thick sections from the basal part of the stem, using three replicates per treatment and five cross-sections per treatment per replicate. Healthy-looking tissues of infected plants were harvested at the end of each experiment (6 weeks after inoculation). Tissues from various parts of infected and control plants were used at the end of the experiment to determine the degree of $V$. dahliae colonization, using both classical (i.e., microsclerotia under the microscope, reisolation) and molecular methods, using the PCR protocol described by El Hadrami and associates (2007), with a slight modification. Briefly, 200 to $500 \mathrm{mg}$ of tissues from the lower, middle, and upper portions of the plant were sampled and were subjected to a DNA extraction and semiquantitative PCR, using primers for the constitutively expressed $\beta$-tubulin and 18S genes (Vd-bTubF: 5'-GTCGACCAGGTTCTCGATGT-3'; Vd-bTubR: 5'-AAGCCGACCATGAAGAAGTG-3'; Vd-18SF: 5'-CGGGGAGGTAGTGACGATAA-3'; Vd-18SR: 5'-CATTAC GGCGGTCCTAGAAA-3'). The amplified products were 473 bp for the $\beta$-tubulin (GenBank number VDAG_10074.1) and 407 bp for the 18S (GenBank number AY489705.1). Quantification and limits of detection were determined based on a preestablished dilution series of DNA isolated from the tested isolates, and a final DNA concentration of $10 \mathrm{ng} / \mu \mathrm{l}$ was used in all quantifications. The colonization percentages were calculated taking into account all tested samples from a given plant. Experimental replicates were analyzed separately, and an overall percentage was deduced for each treatment.

\section{Potato soluble phenolics extraction and analysis.}

For extraction, $1 \mathrm{~g}$ of $\mathrm{FW}$ representing a mixture of leaves, petioles, and stems was crushed in a cold mortar containing 1 $\mathrm{ml}$ of $80 \%$ ( $\mathrm{vol} / \mathrm{vol})$ aqueous methanol solution and was analyzed by HPLC, according to the procedure described by El Hadrami and associates (2010). Identification of the major compounds was conducted based on their characteristic features, including absorption spectra, retention times, comparison with our database standards, coelution with commercial standards as well as TLC analyses. Quantitative analyses were performed by HPLC, using the peak area with reference to a standard curve of a commercial rutin solution (Sigma-Aldrich Co., Oakville, Canada). TLC analyses were carried out using a silica gel plate and various solvent systems, i.e., chloroform/methanol/ water (6:3:1, vol/vol/vol), butanol/water/acetic acid (5:4:1, vol/ vol/vol Developed TLC plates were visualized under UV light (254 to $365 \mathrm{~nm}$ ) or by staining with specific reagents, such as the Benedickt reagent (Reznik and Egger 1961). The $R_{\mathrm{f}}$ (retardation factor) of the visualized phenolics, especially rutin, were recorded in coelution and in comparison with commercial standards. Analyses were also carried out using ACQUITY UPLC coupled to a triple quadrupole mass spectrometer (Micromass Canada, Inc., Mission, BC, Canada), according to the procedure described by El Hadrami and Daayf (2009). For rutin analysis, the first and third stages of quadrupole mass analyzers were set to follow the fragmentation transition of the precursor ion at $\mathrm{m} / \mathrm{z}, 611$ to the product ion at $\mathrm{m} / \mathrm{z}, 303$, which was the most abundant isotopic molecular weight. Data were acquired and processed using MassLynx Software's Application Manager v. 4.1. (Waters Inc.).

\section{Effects of rutin on growth and sporulation of $V$. dahliae.}

Phenolic extracts and commercial rutin solutions were dried, the final residue was resuspended in appropriate volumes of $10 \%$ (vol/vol) ethanol and was used for in vitro assays. These assays consisted of petri plates containing water-agar media onto which plugs of two isolates of fresh V. dahliae (Vd-9 and Vd-21) were transferred. Four sterile 5-mm diameter filter paper disks, containing $20 \mu \mathrm{l}$ of either phenolic extracts, various concentrations of commercial rutin $(1.6 \mathrm{nM}$ to $1.6 \mathrm{mM}$ with $10 \times$ increments), $10 \%$ ( vol/vol) ethanol, or water were introduced onto each plate. The diameters of $\mathrm{V}$. dahliae colonies were measured every three days, and their variation over time was used to calculate the area under the growth progressing curve (AUGPC) as follows.

$$
\mathrm{AUGPC}=\sum_{i=1}^{\mathrm{n}-1}\left[\left(\left(y_{i}+y_{i}+1\right) / 2\right)\left(t_{i+1}-t_{i}\right)\right]
$$

where $y i$ is the surface area of the growing colony at the time $t i$. These data were used to generate a linear regression that served to identify $\mathrm{IC}_{50}$ values. A second experiment was set up to determine the inhibition threshold of rutin directly in the media, in which concentrations of 0 to $1.5 \mathrm{mM}$ were tested on both growth and sporulation. To determine the effect of rutin on sporulation, spores produced on cultures grown on media amended with rutin concentrations of 0 to $1.5 \mathrm{mM}$ were collected and counted.

Additional experiments were conducted to examine the effect of supplementation of potato tissues with rutin on further growth and development of V. dahliae in planta. Freshly harvested potato (cv. Russet Burbank) leaves, stem sections, and roots were washed under running distilled water, were surface-sterilized in 5\% bleach, and were rinsed in SDW before being placed on wet sterile filter paper in petri dishes. These tissues were then infiltrated with a $100-\mu l$ solution of commercial rutin dissolved in water and calibrated at $75 \mu \mathrm{g} / \mathrm{ml}$. Half the replicates were kept as controls, while the other half were further inoculated with $V$. dahliae. Control plant tissues were infiltrated with water. The inoculum was prepared from grown colonies of isolates $\mathrm{Vd}-9$ and $\mathrm{Vd}-21$, according to the procedure described above. Inoculations were made by spreading $100-\mu l$ inoculum droplets around the rutin-infiltrated sites. Inoculated and control leaves and stem sections were kept at $20^{\circ} \mathrm{C}$ days and $16^{\circ} \mathrm{C}$ nights with a 16-h photoperiod, while root sections were kept 
at the same temperature in darkness. At 3 and 7 days after inoculation, samples were harvested and were immediately frozen until they were extracted and analyzed. Samples harvested before the treatments were used as background controls for the analysis. Separation of the purified compounds was conducted by TLC developed in chloroform/methanol/water $(6: 3: 1, \mathrm{vol} / \mathrm{vol} / \mathrm{vol})$ and compounds were further stained with $1 \%(\mathrm{wt} / \mathrm{vol})$ vanillin- $\mathrm{HCl}$ or ninhydrin. HPLC analysis was conducted as described above to reveal both qualitative and quantitative changes in potato extracts in response to both infiltration and inoculation with $V$. dahliae isolates.

A further set of experiments was conducted using potato tuber slices. Tubers from cv. Russet Burbank were washed, were surface-sterilized $(0.6 \%$ [wt/vol] sodium hypochlorite solution containing $0.1 \%$ [vol/vol] Tween 20), were dried under a flow hood, and were then thinly sliced using a home chip supreme slicer (Supreme Slicer; StarFrit, Ottawa, Canada). Tuber slices ( 2 to $3 \mathrm{~mm}$ thick) were treated with SDW, 10\% (vol/vol) CMV extract $(0.3 \mathrm{~g} / \mathrm{ml}$ in water), or $1 \mu \mathrm{g} / \mathrm{ml}$ of rutin, quercetin, naringenin, or phloroglucinol. Half the replicates were kept as controls and were mock-inoculated with water, while the other half were inoculated with $200 \mu$ of a $10^{6}$ spore $/ \mathrm{ml}$ conidial suspension of isolate Vd-9. Tuber slice samples were harvested at 3 and 7 days after inoculation and were analyzed by HPLC as described above.

\section{Degradation of rutin and other related phenolics by $V$. dahliae in vitro.}

To test the possible degradation of rutin and other related compounds such as quercetin, naringenin, and phloroglucinol by $V$. dahliae, the selected isolates ( Vd-9 and $\mathrm{Vd}-21$ ) were grown in $100 \mathrm{ml}$ of Czapeck-Dox liquid media (Hurst et al. 2002), either nonamended or amended with $1 \mathrm{ml}$ of commercial standard solutions of the tested compounds calibrated at $100 \mu \mathrm{g} / \mathrm{ml}$ in dimethyl sulfoxide (DMSO). Media amended only with $1 \mathrm{ml}$ of DMSO were used as controls. The inoculated media as well as the controls were kept at room temperature on a shaker in the dark at $120 \mathrm{rpm}$. Samples consisting of $1-\mathrm{ml}$ solutions were taken at $0,3,7,15,21$, and 28 days after transfer. They were immediately analyzed by HPLC or kept at $-20^{\circ} \mathrm{C}$ until needed. Identification of each compound or its product of degradation was based on their characteristics, including absorption spectra, retention times, comparison and coelution with commercial standards, and on TLC and HPLC. Quantitative analyses were performed by HPLC using the peak area with reference to a standard curve made for each commercial standard (Sigma-Aldrich Co.). Three replicates per treatment $\times$ isolate combination were considered for this experiment. Quantifications of the added compounds and their products of degradation were performed in triplicate and the data are reported as the recorded average values.

The products of degradation of rutin and quercetin were further characterized using an ACQUITY UPLC system (Waters Inc.) connected to a triple quadrupole mass spectrometer system Quattro Ultima (Micromass) under the same conditions described above. The first and third stages of the quadrupole mass analyzer were set to follow the fragmentation transition of the precursor ion at $m / z 343.26$ (2-PCPGCA), which was the most abundant isotopic molecular weight, to the product ions at $\mathrm{m} / \mathrm{z}$ 155.03 and 127.03 , representing protocatechuic acid and phloroglucinol, respectively. Data were acquired and processed using MassLynx Software's Application Manager v. 4.1. (Waters Inc.).

\section{Protein extraction and quercetinase activity assay.}

Proteins were isolated from $200 \mathrm{mg}$ of a freshly grown mycelium of $V$. dahliae isolates $\mathrm{Vd}-9$ and $\mathrm{Vd}-21$ in $0.1 \mathrm{M}$ potassium phosphate buffer, $\mathrm{pH}$ 7.0. The quercetinase activity was assayed following the method described by Oka and associates (1971), which monitors the decrease in absorbance at $367 \mathrm{~nm}$ $\left(\mathrm{A}_{367 \mathrm{~nm}}\right)$ as a result of quercetin breakdown $(\varepsilon=20,000 \mathrm{M} / \mathrm{cm})$ by a known volume of enzyme added to a mixture consisting of $0.1 \mathrm{M} 2-(\mathrm{N}$-morpholino)ethanesulfonic acid buffer, $\mathrm{pH} 6.0$, quercetin, and copper. Every assay was repeated five times and the data was expressed as a percentage of the control.

\section{Experimental design and data analyses.}

Experimental trials were conducted in triplicate, according to a randomized complete block design. In each trial, five individual plants were considered for disease assessment and sampling. Quantitative data were used for variance analysis using Statistica software (Statsoft Inc., Tulsa, OK, U.S.A.). Analysis of variance (ANOVA) was followed by pairwise comparison of the means, using the Newman-Keuls test at $P<0.05$. Linear regression analysis was also conducted to determine whether rutin content explains any of the disease severity reduction assessed. In the experiments set to determine the ability of $V$. dahliae to catabolize rutin, quercetin, or naringenin, the data were expressed as a percentage of the initial amount added to the liquid media and arcsine-transformed before use in ANOVA. Rutin effects on pathogen growth and sporulation were conducted at least twice. Quercetinase activity was determined based on five independent assays performed for each treatment. Collected data points were used in a linear regression of the decrease in $\left(A_{367 \mathrm{~nm}}\right)$ over time to determine the enzymatic activity.

\section{ACKNOWLEDGMENTS}

This research was supported by a grant from the Natural Sciences and Engineering Research Council (NSERC-Canada) to F. Daayf. We thank C. Rampitsch and B. McCallum for revising the manuscript. Special thanks to G. Stacey, whose final review and suggestions substantially improved the quality of this manuscript.

\section{LITERATURE CITED}

Alkher, H., El Hadrami, A., Rashid, K. Y., Adam, L. R., and Daayf, F. 2009. Cross-pathogenicity of Vertcillium dahliae between potato and sunflower. Eur. J. Plant Pathol. 124:505-519.

Andersen, Ø. M., and Markham, K. R. 2006. Page 1237 in: Flavonoids: Chemistry, Biochemistry, and Applications. Taylor \& Francis, London.

Arfaoui, A., El Hadrami, A., Mabrouk, Y., Sifi, B., Boudabous, A., El Hadrami, I., Daayf, F., and Chérif, M. 2007. Treatment of chickpea with Rhizobium isolates enhances the expression of phenylpropanoid defense-related genes in response to infection by Fusarium oxysporum $\mathrm{f}$. sp. ciceris. Plant Physiol. Biochem. 45:470-479.

Báidez, A. G., Gómez, P., Del Río, J. A., Ortuño, A. 2007. Dysfunctionality of the xylem in Olea europea L. plants associated with the infection process by Verticillium dahliae Kleb. Role of phenolic compounds in plant defense mechanism. J. Agric. Food Chem. 55(9):3373-3377.

Bailey, K. L., and Lazarovits, G. 2003. Suppressing soil-borne diseases with residue management and organic amendments. Soil Till. Res. 72:169-180.

Barry, K. M., Davies, N. W., and Mohammed, C. L. 2002. Effect of season and different fungi on phenolics in response to xylem wounding and inoculation in Eucalyptus nitens. For. Path. 32:163-178.

Bartz, W. 1971. Über den Abbau aromatischer Verbindungen durch Fusarium oxysporum Schlecht. Arch. Mikrobiol. 78:341-352.

Bauch, J., A. L. Shigo, and M. Starck. 1980. Wound effects in the xylem of Acer and Betula species. Holzforschung 34: 153-160.

Bennet, R. N., and Wallsgrove, R. M. 1994. Secondary metabolites in plant defence mechanisms. New Phytol. 127:617-633.

Braune, A., Gutschow, M., Engst, W., and Blaut, M. 2001. Degradation of quercetin and luteolin by Eubacterium ramulus. Appl. Environ. Microbiol. 67:5558-5567.

Buer, C. S., Muday, G. K., and Djordjevic, M. A. 2007. Long distance transport flavonoids. Plant Physiol. 145:478-490.

Campbell, C. L., and Madden, L. V. 1990. Introduction to plant disease epidemiology, p. 532. New York: Wiley.

Daayf, F., and Lattanzio, V. 2008. Page 416 in: Recent Advances in Poly- 
phenol Research, Vol. 1. Wiley InterScience, New York.

Daayf, F., El Hadrami, A., Adam, L. R., and Ballance, G. M. (eds.) 2006. Polyphenols Communications 2006. XXIII International Conference on Polyphenols, August 22-25, 2006. Winnipeg, Canada.

Daayf, F., Adam L., and Fernando, W. G. D. 2003. Comparative screening of bacteria for biological control of potato late blight (strain US-8), using in-vitro, detached-leaves, and whole plant testing systems. Can. J. Plant Pathol. 25:276-284.

Daayf, F., Schmitt, A., and Belanger, R. R. 1997. Evidence of phytoalexins in cucumber leaves infected with powdery mildew following treatment with leaf extracts of Reynoutria sachalinensis. Plant Physiol. 113:719. 727

Davis, J. R., Huisman, O. C., Westermann, D. T., Hafez, S. L., Everson, D. O., Sorensen, L. H., and Schneider, A. T. 1996. Effects of green manures on verticillium wilt of potato. Phytopathology 86:444-453.

Dixon, R. A., and Paiva, N. L. 1995. Stress-induced phenylpropanoid metabolism. Plant Cell 7:1085-1097.

El Hadrami, A., Adam, L. R., and Daayf, F. 2009a. Study of some commonalities between the action mechanisms of selected green manures used to control potato verticillium wilt. Abstr. Page 86 in: 10th International Verticillium Symposium. November 16-20, 2009. The International Verticillium Steering Committee and the Local Organizing Committee, Corfu, Hellas, Greece.

El Hadrami, A., El Hadrami, I., and Daayf, F. 2009b. Suppression of induced plant defense responses by fungal pathogens. Pages 231-268 in: Molecular-Plant Microbe Interactions. K. Bouarab, N. Brisson, F. Daayf, eds. CAB International, Wallingford, U.K.

El Hadrami, A., Fernando W. G. D., and Daayf, F. 2010. Variations in relative humidity modulate Leptosphaeria spp. pathogenicity and interfere with canola mechanisms of defence. Eur. J. Plant Pathol. 126:187-202.

El Hadrami, A., and F. Daayf. 2009. Priming canola resistance to blackleg with weakly aggressive isolates leads to an activation of hydroxycinnamates. Can. J. Plant Pathol. 31:393-406.

El Hadrami, A., Wally, O., Adam, L. R., and Daayf, F. 2007. PCR-based determination of colonization patterns during potato tuber infection by single and multiple pathogens. Eur. J. Plant Pathol. 117:201-218.

Fravel, D. R., and Larkin, R. P. 2000. Effect of sublethal stresses on microsclerotia of Verticillium dahliae. Pages 309-314 in: Advances in Verticillium: Research and Disease Management. E. C. Tjamos, R. C. Rowe, J. B. Heale, and D. R. Fravel, eds. American Phytopathological Society, St. Paul, MN, U.S.A.

Harborne, J. B., and Williams, C. A. 2000. Advances in flavonoid research since 1992. Phytochemistry 55:481-504.

Hurst, C. J. Crawford, R. L. Knudsen, G. R. McInerney, M. J., and Stetzenbach L. D. 2002. Manual of Environmental Microbiology. ASM Press, Washington, DC.

Kunkel, B. N., and Brooks, D. M. 2002. Cross talk between signaling pathways in pathogen defense. Curr. Op. Plant Biol. 5:325-331.

Lagrange, H., Jay-Allamand, C., and Lapeyrie, F. 2001. Rutin, the phenolglycoside from eucalyptus root exudates, stimulates Pisolithus hyphal growth at picomolar concentrations. New Phytol. 149:349-355.

Mercado-Blanco, J., Rodríguez-Jurado, D., Hervás, A., Jiménez-Díaz, R. M., 2004. Suppression of verticillium wilt in olive planting stocks by rootassociated fluorescent Pseudomonas spp. Biol. Control 30:474-486.

Merkens, H., Sielker, S., Rose, K., and Fetzner, S. 2007. A new mocupin quercetinase of Streptomyces sp. FLA- identification and heterologous expression of the queD gene and activity of the recombinant enzyme towards different flavonols. Arch. Microbiol. 187:475-487.

Ochiai, N., Powelson, M. L., Dick, R. P., and Crowe, F. J. 2007. Effects of green manure type and amendment rate on verticillium wilt severity and yield of Russet Burbank potato. Plant Dis. 91:400-406.

Oka, T., and Simpson, F. J. 1971. Quercetinase, a dioxygenase containing copper. Biochem. Biophys. Res. Commun. 43:1-5.

Oka, T., Simpson, F. J., Child, J. J., Mills, C. 1971. Degradation of rutin by
Aspergillus flavus. Purification of the dioxygenase, querecentinase Can. J. Microbiol. 17:111-118.

Osbourn, A. E. 1996. Performed antimicrobial compounds and plant defense against fungal attack. Plant Cell 8:1821-1831.

Paulin, M. M., Novinscak, A., St-Arnaud, M., Goyer, C., DeCoste, N. J., Privé, J. P., Owen, J., Filion, M. 2009. Transcriptional activity of antifungal metabolite-encoding genes $p h l D$ and hcnBC in Pseudomonas spp. using qRT-PCR. FEMS (Fed. Eur. Microbiol. Soc.) Microbiol. Ecol. 68:212-222.

Phillips, D. A. 1993. Flavonoids-Plant signal to soil microbes. Pages 201-231 in: Recent advances in phytochemistry, Vol. 26. Phenolics metabolism in plants. H. A. Stafford, P. K. Ibrahim. eds. Plenum Press, New York.

Ramos, T., El Bellaj, M., El Idrissi-Tourane, A., Daayf, F., and El Hadrami, I. 1997. Phenolamides of palm rachis, components of defense reaction of date palm against Fusarium oxysporum f. sp. albedinis, the causal agent of Bayoud. J. Phytopathol. 145:487-493.

Rayapuram, C., and Baldwin, I. T. 2007. Increased SA in NPR1-silenced plants antagonizes JA and JA-dependent direct and indirect defenses in herbivore-attacked Nicotiana attenuata in nature. Plant J. 52:700-715.

Reznik, H., and Egger, K. 1961. Benedikts reagens als Indikator für Phenolische Ortho-dihydroxygruppen. Z. Ann. Chem. 183:196-199.

Sariaslani, F. S., and Dalton, H. 1989. Microbial enzymes for oxidation of organic molecules. Crit. Rev. Biotechnol. 9:171-257.

Simpson, F. J., Talbot, G., and Westlake, D. W. S. 1960. Production of carbon monoxide in the enzymatic degradation of rutin. Biochem. Biophys. Res. Comm. 2:15-18.

Spoel, S. H., Koornneef, A., Claessens, S. M. C., Korzelius, J. P., van Pelt, J. A., Mueller, M. J., Buchala, A. J., Métraux, J. P., Brown, R., Kazan, K., Van Loon, L. C., Dong, X., and Pieterse, C. M. J. 2003. NPR1 modulates cross-talk between salicylate- and jasmonate-dependent defense pathways through a novel function in the cytosol. Plant Cell 15:760:770.

Steiner, R. A., Meyer-Klaucke, W., and Dijkstra, B. 2002. Functional analysis of the copper-dependent quercetin 2,3-dioxygenase. 2. X-ray absorption studies of native enzyme and anaerobic complexes with the substrates quercetin and myricetin. Biochemistry 41:7963-7968.

Subbarao, K. V., Kabir, Z., Martin, F. N., and Koike, S. T. 2007. Management of soilborne diseases in strawberry using vegetable rotations. Plant Dis. 91:964-972.

Thatcher, L. F., Manners, J. M., and Kazan, K. 2009. Fusarium oxysporum hijacks COI1-mediated jasmonate signaling in order to promote disease development in Arabidopsis. Plant J. 58:927-939.

Tjamos, E. C., Tsitsigiannis, D. I., Tjamos S. E., Antoniou, P. P., and Katinakis, P. 2004. Selection and screening of endorhizosphere bacteria from solarized soils as biocontrol agents against Verticillium dahliae of solanaceous hosts. Eur. J. Plant Pathol. 110:35-44.

Tranchimand, S., Ertel, G., Gaydou, V., Gaudin, C., Tron, T., and Iacazio, G. 2008. Biochemical and molecular characterization of a quercetinase from Penicillium olsonii. Biochimie 90:781-789.

Treutter, D. 2006. Significance of flavonoids in plant resistance: a review. Environ. Chem. Lett. 4:147-157.

Uppal, A. K., El Hadrami, A., Adam, L. R., Tenuta, M., and Daayf, F. 2008. Biological control of potato verticillium wilt under controlled and field conditions using selected bacterial antagonists and plant extracts. Biol. Control 44:90-100.

Uppal, A. K., El Hadrami, A., Adam, L. R., Tenuta, M., and Daayf, F. 2007. Pathogenic variability of Verticillium dahliae Kleb. isolates from potato fields in Manitoba and screening of bacteria for their biocontrol. Can. J. Plant Pathol. 29:141-152.

Westlake, D. W. S., Roxburgh, J. M., and Talbot, G. 1961. Microbial production of carbon monoxide from flavonoids. Nature 189:510-511.

Wray, J. W., and Abeles, R. H. 1993. A bacterial enzyme that catalyzes formation of carbon monoxide. J. Biol. Chem. 268:21466-21469. 